\title{
Optimisation of metabarcoding for monitoring marine macrobenthos: primer choice and morphological traits determine species detection in bulkDNA and eDNA from the ethanol preservative
}

\author{
Sofie Derycke ${ }^{1}$, Sara Maes ${ }^{1}$, Laure Van den Bulcke ${ }^{1}$, Joran Vanhollebeke ${ }^{1}$, Jan Wittoeck ${ }^{1}$, \\ Hans Hillewaert ${ }^{1}$, Bart Ampe ${ }^{1}$, Annelies Haegeman ${ }^{1}$, Kris Hostens ${ }^{1}$, and Annelies De \\ Backer $^{1}$ \\ ${ }^{1}$ Research Institute for Agriculture Fisheries and Food Research
}

August 5, 2020

\begin{abstract}
DNA metabarcoding is a promising method to increase cost and time efficiency of marine monitoring, providing that the impact of methodological choices on the reliability and reproducibility of results are well understood. Here, we investigated the impact of primer choice, DNA source (bulk DNA or eDNA from the ethanol preservative) and morphological traits (body size and body skeleton) on species detection in four distinct macrobenthos communities from the North Sea. We generated a reference database with COI sequences for macrobenthos from the North sea and applied DNA metabarcoding using five COI primer sets. At most $22 \%$ of the ASVs were assigned taxonomy at the phylum level, despite the availability of a nearly complete reference database. However, the unassigned ASVs represented only a small fraction of the total reads (13\%). The Leray primer set outperformed the four other primer sets in the number of non-chimeric reads and species detected, and in the recovery of beta diversity patterns. Community composition differed significantly between bulk DNA and eDNA samples, but both sample types were able to differentiate the four communities. Importantly, the probability of detecting a species in the eDNA from the ethanol preservative was significantly lower than for bulk DNA for macrobenthos species with small to medium body size and for species with chitine or $\mathrm{CaCO} 3$ in their skeleton. Detection in the bulk DNA samples was not affected by the traits investigated, indicating that monitoring of macrobenthos species will be most robust when using bulk DNA as template for metabarcoding.
\end{abstract}

\section{INTRODUCTION}

Marine ecosystems deliver many important services to society such as climate regulation, coastal protection, food provision and recreation (Barbier, 2017; Duncan, Thompson, \& Pettorelli, 2015). To maintain ecosystem services, good ecosystem health and proper ecosystem functioning are crucial (Barbier, 2017) and both are inextricably linked to marine biodiversity (Goodwin et al., 2017; Solan et al., 2004). Several legal and policy frameworks have been developed with conservation of marine ecosystem health as main objective (Goodwin et al., 2017; Halpern et al., 2015) and which include monitoring of benthic diversity. The characterization of benthic diversity relies mostly on morphological taxonomy, which is slow and expensive because of manual sorting and visual identification of taxa in the samples. The development of innovative, costeffective monitoring tools that allow a quick screening of benthic diversity in large marine areas is therefore needed (Borja et al., 2016; Elliott et al., 2018).

DNA metabarcoding is a promising tool for monitoring benthic environments (Aylagas, Borja, Irigoien, \& Rodriguez-Ezpeleta, 2016; Aylagas, Borja, Muxika, \& Rodriguez-Ezpeleta, 2018; Elbrecht, Vamos, Meissner, 
Aroviita, \& Leese, 2017; Leray \& Knowlton, 2015). Instead of identifying all specimens morphologically, DNA is extracted from the total community, a short fragment of the genome is amplified through PCR, the resulting library is sequenced using high throughput sequencing and finally, the resulting sequences are bioinformatically processed (Pawlowski et al., 2018). Each of these steps can introduce bias and errors (Alberdi, Aizpurua, Gilbert, \& Bohmann, 2018), and optimization of the methodological aspects of the protocol are needed to achieve reliable, comparable and repeatable data across studies. Different marker genes can yield very different diversity estimates of invertebrate communities and combining different markers increases the detection of all species (Alberdi et al., 2018; Marquina, Esparza-Salas, Roslin, \& Ronquist, 2019). For metabarcoding metazoan communities, the mitochondrial COI gene is the preferred marker gene because of the availability of large reference databases and the presence of sufficient genetic variation to allow species level identification (Andujar, Arribas, Yu, Vogler, \& Emerson, 2018). The final detection of a particular species in the sample greatly depends on its biomass and on the primers used in the DNA metabarcoding protocol (Elbrecht, Peinert, \& Leese, 2017). The PCR step is one of the most influential steps for the detection of species using DNA metabarcoding, where primer mismatches, GC content, template switching, stochasticity and polymerase errors can affect the number of sequence variants found in the dataset (Kebschull \& Zador, 2015). Different primer sets are available for the COI gene which also leads to differences in diversity (Braukmann et al., 2019; Elbrecht \& Leese, 2017; Lobo, Shokralla, Costa, Hajibabaei, \& Costa, 2017). Consequently, it is important to investigate primer performance for (bio)geographic areas and taxonomic groups that have not been studied before to obtain accurate results with DNA metabarcoding.

Next to primer choice, the DNA source used in metabarcoding studies can affect whether or not a species is detected. DNA can be extracted from bulk specimens that have been separated from the sediment by sieving, decantation and manual sorting (Aylagas, Mendibil, Borja, \& Rodriguez-Ezpeleta, 2016) or from the ethanol preservative in which the sieved macrobenthos sample was preserved (Zizka, Leese, Peinert, \& Geiger, 2019). Bulk DNA samples, can further be sorted in different size fractions to enhance detection of smaller sized animals (Elbrecht, Peinert, et al., 2017; Leray \& Knowlton, 2015) which comes at an extra cost of time to process samples. Extracting DNA from the ethanol fixative avoids the time consuming step of having to sort specimens and has the added advantage that voucher specimens are still present after the analyses. However, non-target species such as bacteria and fungi can be encountered more when analyzing the ethanol preservative compared to bulk samples (Gauthier et al., 2020). A comparison between bulk and ethanol samples for freshwater invertebrates showed that the two approaches yield very different community compositions (Marquina, Esparza-Salas, et al., 2019; Zizka, Leese, et al., 2019). Nevertheless, DNA metabarcoding of the ethanol fraction has also shown considerable overlap with morphology based analyses of freshwater communities illustrating its potential for monitoring studies (Martins et al., 2019). In view of the above mentioned advantages when using the ethanol preservative, understanding the different results between bulk DNA and eDNA from the ethanol preservative is essential to determine their applicability for monitoring studies. For instance, it has been shown that small and weakly sclerotized freshwater insects species are overrepresented in the ethanol preservative while large and strongly sclerotized species are overrepresented in the bulk DNA (Marquina, Esparza-Salas, et al., 2019). Consequently, morphological traits of species may be important to explain their detection in DNA metabarcoding studies but this link has hitherto not been investigated for marine macrobenthos.

In this study, we sampled four distinct macrobenthos communities in the North Sea and identified them using traditional morpho-taxonomy before molecular processing. We generated $104 \mathrm{COI}$ reference sequences from macrobenthos species in the Belgian part of the North Sea to allow taxonomic assignment of the metabarcode datasets. Our first aim was to find the best primer set to characterise marine macrobenthos communities through DNA metabarcoding. We tested five primer sets from the literature and defined the best primer set as the set that detected the highest number of morphological species and that was able to differentiate macrobenthic communities. Second, we investigated whether morphological and metabarcode analyses detected the same species. We expected to detect more diversity in the metabarcode datasets compared to the morphological dataset as specimens are not lost during the sorting process. We also expected to find a high proportion of species shared between the two approaches. Third, we evaluated whether eDNA 
from the ethanol preservative could be used for monitoring by comparing its alpha and beta diversity patterns with that of bulk DNA. Based on the results in freshwater communities, we expected to find beta diversity patterns that differentiate the four communities in the bulk DNA and eDNA from the ethanol preservative and to find additional non-macrobenthic taxa in the eDNA from the ethanol preservative. Fourth, we investigated whether morphological and ecological traits of the macrobenthos species could explain their probability of detection in the bulk DNA and eDNA from the ethanol preservative. Based on the results of insects in freshwater environments (Marquina, Esparza-Salas, et al., 2019), we expected to find a higher probability of detecting small and weakly sclerotized species in the ethanol preservative.

\section{MATERIAL AND METHODS}

We evaluated the ability of DNA metabarcoding to detect marine macrobenthos species from the Belgian part of the North sea, a relatively small area $\left(\right.$ ca $3600 \mathrm{~km}^{2}$ ) subjected to many human activities (Douvere, Maes, Vanhulle, \& Schrijvers, 2007). The area has been extensively monitored for almost 20 years, resulting in well described macrobenthos communities (Breine et al., 2018; Degraer et al., 2008; Van Hoey, Degraer, \& Vincx, 2004).

\section{Sample collection}

Samples were collected in the Belgian part of the North Sea in autumn 2017 in four different habitats each characterized by a distinct macrobenthic community (Fig 1): 1/ a fine muddy habitat with low diversity and low abundances with mainly sessile burrowing dwelling species (Limecola balthica community, ZVL); 2/a coastal fine sand habitat, characterized by high diversity and high abundances with mainly sessile, tube building burrowers (Abra alba community, 120), 3/ a medium sandy habitat with low taxon richness and low abundances (Nephtys cirrosa community, 330) and 4/ an offshore coarse sandy habitat, with medium diversity and low abundances (Hesionura elongata community, 840). The latter two habitats are characterized by relatively small, free-living and burrowing macrobenthic species. In each of the four communities, three replicate Van Veen grabs (A,B and C) were collected on board of the RV Belgica. The sediment was sieved on board on a $1 \mathrm{~mm}$ sieve. All material (macrobenthos specimens + residue) that remained on the sieve was fixed in ethanol and stored at $-20^{\circ} \mathrm{C}$ until further processing.

\section{Sample processing}

After storage of ca. 8 months at $-20^{\circ} \mathrm{C}$, the 12 samples were shaken and mixed with a spoon to suspend DNA, after which two times $200 \mathrm{ml}$ ethanol were filtered using a $100 \mathrm{ml}$ microfunnel unit with a $0.45 \mu \mathrm{M}$ GN6 Metricel membrane. Filters were stored at $-80^{\circ} \mathrm{C}$. Subsequently, bulk samples were processed largely following the protocol outlined by Aylagas, Mendibil, et al. (2016). In short, samples were six to 13 times decanted in the lab using tap water. Decanting was repeated until no animals were recovered from the samples. All animals remaining on a $1 \mathrm{~mm}$ sieve were collected and stored in ethanol. The remaining material after decantation was sorted in a cleaned tray and heavier animals such as bivalves were picked with clean tweezers and added to the decanted material in ethanol. From each of the four locations, one replicate was identified morphologically (ZVL-A, 120-B, 840-C, 330-C) to the lowest taxonomic level possible prior to grinding. Specimens were identified up to species level, except for juveniles which were identified to the genus level and specimens belonging to Nemertea, Anthozoa and Oligochaeta, which were only identified up to phylum, class and order level, respectively, following the certified protocol for macrobenthos identification (according to ISO16665:2014 and NMBAQCS's "Guidelines for processing marine macrobenthic samples: a Processing Requirements Protocol" Version 1.0). In total, 24 samples were available for further molecular processing (12 ethanol samples and 12 bulk samples).

\section{DNA extraction}

For the ethanol samples, DNA extractions were performed using the Promega Wizard@SV Genomic DNA Purification System. One filter was cut into six pieces and separately lysed overnight at $55^{\circ} \mathrm{C}$ in $275 \mu l$ lysis buffer. The lysate of two pieces was pooled and the three pooled lysates were processed separately using the manufacturer's protocol. This resulted in three DNA extracts of $50 \mu \mathrm{l}$ from one filter for each ethanol 
sample, which were pooled together. Half of the pooled DNA extract $(75 \mu \mathrm{l})$ underwent cleanup using the Promega Wizard@ DNA Clean-Up System and was eluted in 50ul TE-buffer following the manufacturer's protocol.

For the bulk samples, specimens and ethanol were mixed into a homogeneous solution using a blender or, in case less than $100 \mathrm{ml}$ sample was available, grinded with mortar and pestle. Depending on the volume of the sample, two (for samples $<100 \mathrm{ml}$ ) or six (for samples $>100 \mathrm{ml}$, this was the case for the three replicates of station 120) subsamples of $2 \mathrm{ml}$ were transferred to an Eppendorf tube for DNA extraction. The remaining "soup" was stored at $-20^{\circ} \mathrm{C}$. The Eppendorf tubes with the $2 \mathrm{ml}$ "soup" were centrifuged and the supernatant was removed. To remove the remaining ethanol, the Eppendorf tubes were incubated at $50^{\circ} \mathrm{C}$ with open lid until the pellet was dry. The pellet was then used to extract DNA using the DNeasy PowerSoil Kit (QIAGEN) following the manufacturer's protocol. The lysis step was done by adding $10 \mu \mathrm{l}$ of Proteinase $\mathrm{K}(20 \mathrm{mg} / \mathrm{ml})$ and incubating overnight in a thermal shaker at $56^{\circ} \mathrm{C}$ and $1000 \mathrm{rpm}$. The same volume of each subsample was pooled to obtain an end volume of $50 \mu \mathrm{L}$, which was then cleaned with the Wizard DNA Clean-Up System (Promega) according to manufacturer's protocol. From each of the 12 bulk samples, one DNA extract of $50 \mu \mathrm{L}$ was obtained.

\section{Library preparation}

We used a two step amplification protocol, which has been shown to be a robust and cost effective method for DNA metabarcoding (Zizka, Elbrecht, Macher, \& Leese, 2019). Each of the 24 DNA extracts (12 from ethanol and 12 from bulk) were PCR amplified in triplicates. The PCR mix was prepared in $25 \mu$ reactions consisting of $12.5 \mu \mathrm{l} 2 \mathrm{x}$ KAPA HiFi Hotstart ReadyMix, $0.75 \mu \mathrm{l}$ of forward and reverse primer $(10 \mu \mathrm{M}), 8.5$ $\mu \mathrm{l}$ nuclease free water and $2.5 \mu \mathrm{l}$ of DNA from the bulk samples. For the eDNA samples from the ethanol preservative, the same mix was prepared but with $6 \mu \mathrm{l}$ nuclease free water and $5 \mu \mathrm{L}$ of eDNA. PCR cycling conditions were: initial denaturation for $3 \mathrm{~min}$ at $95^{\circ} \mathrm{C}, 35$ cycles of denaturation for $30 \mathrm{~s}$ at $98^{\circ} \mathrm{C}$, annealing for $30 \mathrm{~s}$ at $57{ }^{\circ} \mathrm{C}$ and extension for $30 \mathrm{~s}$ at $72{ }^{\circ} \mathrm{C}$ and a final extension for $1 \mathrm{~min}$ at $72^{\circ} \mathrm{C}$. The three PCR replicates were pooled and $37.5 \mu \mathrm{l}$ of the pooled PCR product was purified using $30 \mu \mathrm{l}$ CleanNGS beads (GC Biotech) according to the manufacturer's protocol and eluted in $40 \mu \mathrm{L}$ TE-buffer. We screened the literature to identify primers that have been successfully used in metabarcoding studies for marine species (Table 1). These primer sets were first tested in silico using EcoPCR (Ficetola et al., 2010) and the MIDORI_UNIQUE_COI_MARINE_20180221 reference dataset (Machida, Leray, Ho, \& Knowlton, 2017) (maximum errors set at 3 , minimum length of the amplicon set at 100, maximum length of the amplicon set at 900). Six primer sets were then tested in the wetlab, and five primer sets yielded a PCR product for our macrobenthos samples (Table 1). Four primer sets (A,B,C and E) amplify the 3' region and one primer set (D) targets the 5' region of the Folmer region (Folmer, Black, Hoeh, Lutz, \& R, 1994) (Table 1). All 24 samples were amplified with these five primer sets. The purified PCR product was then used as template for the index PCR using the Nextera XT Index kit v2 from Illumina. The reaction mix consisted of $5 \mu \mathrm{l}$ nuclease free water, $12.5 \mu \mathrm{l} 2 \mathrm{X}$ KAPA HiFi HotStart ReadyMix, $2.5 \mu \mathrm{l}$ of each index primer $(10 \mu \mathrm{M})$ and $2.5 \mu \mathrm{L}$ purified PCR product. The PCR cycling conditions were: initial denaturation for $3 \mathrm{~min}$ at $95^{\circ} \mathrm{C}, 8$ cycles of denaturation for $30 \mathrm{~s}$ at $95{ }^{\circ} \mathrm{C}$, annealing for $30 \mathrm{~s}$ at $55{ }^{\circ} \mathrm{C}$ and extension for $30 \mathrm{~s}$ at $72{ }^{\circ} \mathrm{C}$ and a final extension for $5 \mathrm{~min}$ at $72^{\circ} \mathrm{C}$. Indexed PCR products were purified with Clean NGS beads. Successful indexing was checked by loading PCR1 and index PCR products on the Qiaxcel (Qiagen). Indexed PCR products were measured twice with the Quantus and equimolarly pooled. The library was sequenced on two Illumina Miseq runs (300bp, PE, sequenced by Admera Health Biopharma Services): primer sets A-D were run together, while primer set E was added to a second run containing the same macrobenthos samples for a study on the variation between biological, DNA and PCR replicates (Van Den Bulcke et al, in preparation). For both runs, 20\% PhiX was added.

\section{Bioinformatic processing}

Demultiplexed reads were provided by the sequencing company (Admera health Biopharma Services) and were checked for quality using MultiQC (Ewels, Magnusson, Lundin, \& Kaller, 2016). Forward and reverse primers were removed using Trimmomatic (Bolger, Lohse, \& Usadel, 2014). Reads were further processed 
using the Dada2 pipeline in the Dada2 v1.12.1 package (Callahan et al., 2016) in R v3.6.2 (Team, 2019) to obtain amplicon sequence variants. Standard filtering parameters were used, except for the maximum number of errors allowed in a read, which was set at 3 . Reads were further trimmed to remove parts with a quality score $<30$ while keeping at least 5 bp overlap. For each sample and each primer set, unique reads were determined, merged and filtered for chimera's. Taxonomy was assigned using a custom made reference database containing in house and Bold COI sequences from macrobenthic species that have been found during monitoring campaigns in the Belgian part of the North Sea over the last ten years. This reference database contained 346 Sanger COI sequences from 306 species. The newly generated COI sequences have been uploaded to BOLD and are part of a larger study to build a COI reference database for macrobenthos from the whole North Sea region (https://northsearegion.eu/geans/). Taxonomy was assigned with the naïve Bayesian classifier (Wang, Garrity, Tiedje, \& Cole, 2007) with the number of bootstraps set at 80 (minBoot $=80)$. Barplots were created in R to visualize the number of reads, ASVs and the percentage of ASVs with assigned taxonomy for the ethanol and bulk samples for each primer set. ASVs that did not receive a taxonomic assignment at the phylum level using our custom reference database were extracted and taxonomic assignment was repeated as above but now using the MIDORI_UNIQUE_COI_MARINE_20180221 reference dataset (Machida et al., 2017) downloaded fromhttp://genoweb.toulouse.inra.fr/frogs_databanks/assignation/to ensure that the lack taxonomic assignment was not caused by the reference database used. Only a small fraction of the data was additionally assigned taxonomy when using the Midori dataset (see results), so all further comparisons were made using taxonomic assignments with our custom made reference database because it has been shown that smaller training datasets tailored to the taxa and geographic region of interest yield better results for genus and species level assignments than using the largest possible database (Macheriotou et al., 2019; Ritari, Salojarvi, Lahti, \& de Vos, 2015). For primer set A, unassigned ASVs after MIDORI were matched against the nt database of NCBI using Blastn to check whether these unassigned ASVs were from non-metazoan origin. Taxonomic assignments with qcov $>50$ and pident $>90$ were considered a reliable hit.

\section{Comparison of species composition between morphology, bulk DNA and eDNA datasets}

The number of unique and shared species for the morphological, bulk and ethanol datasets was evaluated using the unrarified datasets from the four samples that had been identified morphologically and using the UpsetR package(Conway, Lex, \& Gehlenborg, 2017). For the morphological dataset, only specimens identified up to species level were included in the dataset (juvenile identifications to genus, order or phylum level were removed from the dataset). For each of the five primer sets, the total number of species found for each of the four samples was determined and visualized in barplots using $\mathrm{R}$.

\section{Comparison of beta diversity patterns between bulk DNA and eDNA datasets of the five primer sets}

To compare species community composition of the bulk and ethanol samples across primer sets and locations, we rarified all samples in the five datasets at 25000 reads. Samples with less than 25000 reads were removed from the dataset. This threshold was chosen because the plateau phase of the rarefaction curves was reached for all primer sets while a minimum of samples had to be removed $(6,1,2,4$ and 1 sample were removed for primer sets A, B, C, D and E respectively). The resulting count tables were fourth root transformed, a transformation commonly used for ecological datasets with many zeros and a few large values (Quinn \& Keough, 2002). Bray-Curtis dissimilarity index was calculated between samples and used to construct a metric multidimensional scaling (MDS) plot for all primer sets combined and for each primer set separate in the R package Vegan 2.5.6 (Dixon, 2003). Next to this abundance based index, the Jaccard similarity index was calculated for each primer set separate. This index is based on presence/absence data, and determines the percentage of shared species between samples.

Each of the five primer datasets was characterized by a fully crossed design for the factors location (four levels: 120, 330, 840 and ZVL) and DNA source (two levels: eDNA from ethanol and bulk DNA). A two-way PERMANOVA was performed with 9999 permutations to test for significant differences in macrobenthos communi ties between locations, between DNA source and the interaction location X DNA source. The homogeneity of multivariate dispersions for the main factors location and DNA source and their interaction 
was assessed using the betadisper and the permutest functions (9999 permutations) from the Vegan package. Pairwise posthoc tests were conducted for the factor "Location" when the interaction term was non-significant using the pairwiseAdonis library (Martinez Arbizu, 2019). When the interaction term is significant, pairwise tests should be conducted within each level of the factor DNA source. However, in view of the low level of replication at this level, such analyses were inappropriate. The false discovery rate, the expected proportion of false discoveries amongst the rejected hypotheses was controlled by the BH method (Benjamini \& Hochberg, 1995) using 999 permutations. The statistical analyses were conducted on the Bray Curtis and the Jaccard distance matrices for each primer set.

\section{Investigation of traits that impact the probability of detection of macrobenthos in bulk DNA and eDNA}

To determine whether the detection of macrobenthos species in bulk DNA and eDNA datasets could be linked to morphological and biological traits of the species, four categorical traits were scored: body size $(<$ $20 \mathrm{~mm}, 20-100 \mathrm{~mm},>100 \mathrm{~mm}$ ), body skeleton (chitin, $\mathrm{CaCO}_{3}$ or soft tissue), larval stage (benthic or pelagic) and longevity $(<1,1-3,3-10,>10$ years) based on the species-trait dataset of Breine et al. (2018), and for body skeleton based on Brusca and Brusca (2002) and Motokawa (1984). To investigate how these four traits affect the probability of detection (presence/absence) of the species in bulk DNA and eDNA samples, we used a generalized linear mixed effects model approach with the glmer function in the lme4 package (Bates, Maechler, Bolker, \& Walker, 2015). The mixed logistic regression model was built with sample type (bulk DNA or eDNA) and the four traits as fixed effects, while Sample ID was considered as a random effect because eDNA from the ethanol preservative and bulk DNA came from the same sample. We used a logitlink and assumed a binomially distributed error for the presence/absence response variable. We started with simple models always including sample type and the interaction of sample type with one of the four traits. Only traits with significant terms were then used to build more complex models. The final model was chosen based on the lowest AIC and the lowest number of parameters. This model included sample type, body size and body skeleton along with the two way interaction terms with sample type. The significance level of the Type III tests of the fixed model terms was generated using the Anova function of the car package (Fox \& Weisberg, 2019). To visualize the effects of the model terms, least squares means of the fitted model were calculated using the lsmeans function of the emmeans package v 1.4.7 (Lenth, 2020). The obtained probabilities were visualised using ggplot. Posthoc pairwise significance testing for differences between body sizes within sample type and between body skeleton categories within sample type were conducted using the lsmeans function. We restricted the trait analyses to the dataset of primer set A, because this primer set detected most of the morphological species (see results).

\section{RESULTS}

\section{Bioinformatic processing}

The total number of sequences generated for each primer set ranged between 2786114 and 3845487 (ESM Table 1). One sample (ZVLA_B) received very low read numbers for all primer sets. Primer set B yielded the highest number of raw reads, but almost half of the reads were lost during the filtering step. For primer sets $\mathrm{A}$ and $\mathrm{E}$, relatively few reads were lost during filtering, denoising and chimera removal, resulting in more than 2.5M reads for further processing (ESM Table 1, ESM fig 1). Read numbers were comparable between bulk and ethanol samples for each primer set except for primer set A, where approximately three times more reads were obtained for the bulk samples compared to the ethanol samples (ESM Fig2A). Nevertheless, a comparable number of ASVs was obtained for the ethanol and bulk samples with primer set A (average of 149 and 132 respectively, ESM table 1, ESM Fig2B). For primer sets C and E, more ASVs were found in the ethanol than in the bulk samples (ESM Fig2B). The total number of ASVs generated across the 24 samples substantially varied between primer sets and was lowest for primer sets A and E (2139, 22151, 14813, 15211 and 5230 ASVs for primer sets A, B, C, D and E, respectively) (ESM Fig3).

The percentage of ASVs that were assigned taxonomy using our custom COI reference database was low $(22.6 \%, 12.1 \%, 11.7 \%, 4 \%$ and $10.9 \%$ for primer sets A,B,C,D and E respectively; ESM Table 2). However, 
for primer set A, the 1655 unassigned ASVs represented only $13.4 \%$ of the total number of non-chimeric reads generated for that primer set. This percentage was considerably higher for the other primer sets and ranged between 38.4 and $81.7 \%$ (ESM Table 2). Phylum level assignments were comparable between the ethanol and bulk samples for primer sets B, C and D, while more ASVs from the bulk samples were assigned to phyla compared to the ethanol samples for primer sets A (bulk: 30\%, ethanol: 20\%) and D (bulk: 20\%, ethanol: $9 \%$ ) (Fig 2). At the species level, taxonomic assignment of the bulk samples was highest for primer set A (25\%, 9 $\%, 10 \%, 3 \%$ and $16 \%$ for primer sets A,B,C,D and E, respectively). When using the COI Midori dataset for taxonomic assignment of the unassigned sequences, only a small fraction were assigned at the phylum level $(8.6 \%, 0.6 \%, 1.1 \%, 5.7 \%$ and $5.8 \%$ for primer sets A, B, C, D and E, respectively), and these assigned ASVs represented $5.3 \%, 0.5 \%, 0.8 \%, 6.0 \%$ and $20.6 \%$ of the total non-chimeric reads for primersets A,B,C,D and E, respectively. For primer sets A and E, most reads were assigned to the cnidarian Obelia bidentata (1.3\% and $5.1 \%$, respectively) which was found in all ethanol samples, in all bulk samples of location 120 and 330 and in very low abundance in one bulk sample of location 840 (37 and 22 reads, respectively). A detailed list of all species detected after taxonomic assignment with the Midori dataset for each primer set is available in ESM Table 3. To investigate whether the unassigned ASVs after Midori were of non-metazoan origin, a blastn search was done for primer set A against the nt database of NCBI. This resulted in only 83 of the 1471 unassigned ASVs recieving a reliable assignment (query coverage $>50, \%$ identity $>90$ ), representing 58 species. All species had low read numbers, except for Limecola balthica, which was detected in the bulk DNA of two replicates of the Limecola balthica community (ZVL) with more than 10000 reads (ESM Table 4). The non-metazoan taxa were represented by three fungal, two bacterial, five Viridiplantae and 27 algal or diatom species which all together only represented $0.3 \%$ of the total non-chimeric reads (ESM Table 4).

\section{Comparison of species composition between morphology, bulk DNA and eDNA datasets}

Morphological identification of the four samples yielded a total of 57 macrobenthos species. In addition, juveniles of Ophiura,Echinoidea, Ophelia, Nephtys , Phoronis ,Pontocrates , Spio, Magelona , Cirratulidae, Glycera, Lanice as well as specimens belonging to Nemertea, Oligochaeta and Anthozoa were identified to the respective order, genus or phylum. A detailed list of all taxa and their abundance in each location is presented in ESM Table 5. The number of morphological species was highest in Location 120, where 39 species were identified, and decreased over Locations 330, 840 and ZVL (13, 10 and 3, respectively) (ESM Fig 4). For six morphologically identified species (Capitella minima, Caulleriella alata, Mediomastus fragilis, Pseudopolydora pulchra, Spirobranchus lamarckii and Tanaissus liljeborgi ), no COI sequence was present in our reference COI database. For the bulk DNA datasets, all primer sets except primer set D showed the same decreasing trend in species numbers from Location 120, over 330, 840 and ZVL (ESM Fig 4). For the eDNA samples from the ethanol preservative, primer sets B and D identified very low species numbers, and for all primer sets the decreasing trend in species numbers was less clear compared to the bulk DNA dataset. Bulk DNA generally detected a higher number of species than the eDNA samples from the ethanol preservative $(52,37,37,25$ and 44 species for bulk DNA and 45, 13, 22, 4 and 46 species for eDNA from the ethanol preservative with primer sets A, B, C, D and E, respectively). The biggest discrepancy between primer sets was situated in the number of species detected for the most diverse sample 120B (ESM Fig 4).

Of the 57 species that were identified morphologically, primer set A was able to pick up the highest number of species (Fig 3): 34 and 23 of the morphological species were detected in the bulk DNA and in the eDNA from the ethanol preservative, respectively. All primer sets missed a substantial portion of the morphologically identified species: 21,34, 33, 40 and 25 species of the 57 morphological species were only found in the morphological dataset, for primer sets A, B, C, D and E respectively (Fig 3). The number of morphological species detected in the bulk DNA samples (34, 23, 24, 17 and 28 for primer sets A, B, C, D and E respectively) was substantially higher than the number of morphological species detected in the eDNA samples from the ethanol preservative (23, 7, 9, 3 and 22 for primer sets A, B, C, D and E, respectively). Primer sets A and E clearly outperformed the detection of the morphological species in the eDNA from the ethanol preservative.

Comparison of beta diversity patterns between bulk DNA and eDNA datasets of the five primer sets 
The MDS plots illustrate that primer sets A and E have very similar community patterns, while primer sets B, C and D are similar to each other but clearly distinct from the other two primer sets (ESM Fig 5A). All five primer sets were able to differentiate the most diverse location (120) from the three other locations (ESM Fig $5 \mathrm{~B}$ ), but differences in the ability to differentiate the three other locations were observed between primer sets (Fig 4). MDS plots for the five primer sets separately showed that the offshore sandy locations with intermediate diversity (330 and 840) were more similar to each other than to the fine sandy (120) and fine muddy (ZVL) coastal locations (Fig 4). For primer sets A and E, all four locations were recovered as separate clusters when using Bray Curtis dissimilarity index (Fig 4). This separation was less clear when using the Jaccard index (ESM Fig 6). Permanova results were largely consistent across primer sets and between Bray Curtis and Jaccard distance metrics and showed highly significant differences between locations, between bulk DNA or eDNA and for primer sets B, C and D also the interaction effect was highly significant (Table 2). Observed differences between primer sets were the non-significant interaction term "Location x DNA source" for primer sets A and E when the Bray Curtis index was used and a non-significant "DNA source" effect and interaction term "Location x DNA source" for primer set A when the Jaccard index was used (Table 2). Nearly all permdisp results were significant for all primer sets and for both distance metrics (Table 2), indicating that the significant Permanova results were caused by both location effects and dispersion effects. Despite the differences in dispersion of the replicates within sampling locations, the MDS plots show a clear distinction between sampling locations which was supported by a significant main effect of location in Permanova (Table 2) as well as significant posthoc test results for all pairwise combinations of locations for primer sets A and E (ESM Table 6). Permanova revealed significant differences in community structure between bulk DNA and eDNA samples for all primer sets (Table 2).

\section{Morphological traits and community composition of bulk DNA and eDNA from the ethanol preservative}

A detailed comparison between communities recovered in bulk DNA and eDNA from the ethanol preservative was conducted for primer set A. Both DNA datasets detected more species than the morphology based identification (69 species in bulk DNA, 64 in eDNA and 57 in morphology, ESM Fig 7). Although community composition significantly differed between bulk DNA and eDNA of the ethanol preservative (Table 2), 49 species were also shared between the two types of sample (ESM Fig 7). Nevertheless, the differences observed between the two sample types could be explained by morphological traits of the macrobenthos species. The two biological traits larval stage and life span did not result in significant terms and were not included in the final model. The final model included fixed effects of sample type, body size and body skeleton, as well as the two way interaction terms sample type ${ }^{*}$ body size and sample type ${ }^{*}$ body skeleton. The probability of detecting a species in bulk DNA or eDNA from the ethanol preservative was differently affected by body size and body skeleton as shown by the significant sample type * body size and sample type * body skeleton interaction effects (Table 3). The probability of detecting species in the bulk samples was overall high (Fig 5 ), with no significant differences between body sizes and body skeleton (all pairwise comparisons had $\mathrm{p}$ $>0.05$ ). In contrast, the probability of detecting species in the eDNA from the ethanol preservative was significantly higher for soft bodied species compared to species with chitine $(\mathrm{p}=0.0001)$ or CaCO3 in their skeleton $(\mathrm{p}=0.0001)$. The probability of detecting species with small and medium body sizes was significantly higher in bulk DNA samples than in the eDNA samples from the ethanol preservative (body size 0-20: $\mathrm{p}<0.0001$; body size 21-100: $\mathrm{p}=0.003$; Fig 5 ), while the probability of detecting species with large body sizes was not significantly different between the two sample types $(\mathrm{p}=0.18)$. The presence of body skeleton had also a clear impact on the probability of species detection in the two sample types: the probability of detecting species with chitine or $\mathrm{CaCO} 3$ in their skeleton was significantly lower in the eDNA of the ethanol preservative compared to the bulk DNA samples $(\mathrm{p}=0.0002$ and $\mathrm{p}<0.0001$, respectively, Fig 5), while no significant difference was observed between the two sample types for species with soft body skeleton $(\mathrm{p}=0.21)$.

\section{DISCUSSION}

Our focus on well characterised macrobenthic communities from the North sea allowed to investigate and 
understand the biases inherent to DNA metabarcoding when characterising macrobenthic diversity. We found considerable differences in alpha and beta diversity patterns between the tested primer sets and between the two sample types (bulk DNA vs eDNA from the ethanol preservative) and show that the detection of macrobenthic species in the eDNA of the ethanol preservative is linked to their body size and body skeleton. In addition, the one to one comparison between morphology based identification and DNA metabarcoding of bulk DNA and eDNA from the ethanol preservative and the availability of a nearly complete reference database shows that discrepancies between morpho-taxonomic and DNA metabarcode datasets are not entirely the result of incomplete reference databases.

\section{A large fraction of ASVs does not receive taxonomic assignment, despite the well-known species composition in the samples}

Previous COI metabarcode datasets derived from macrobenthos communities have shown that only a small fraction of OTUs receives taxonomic assignment linked to macrobenthos (Aylagas et al., 2018; Leray \& Knowlton, 2015) which has been explained by the incompleteness of reference databases (Leray \& Knowlton, 2015; Wangensteen, Palacin, Guardiola, \& Turon, 2018). Our datasets also contained a low percentage of ASVs (4-22.6\%) with taxonomic assignment. Even when using the MIDORI reference database with almost 47000 marine metazoan species for taxonomic assignment, only an additional $0.6-8.6 \%$ of the ASVs were assigned. Previous macrobenthos metabarcode studies reported a high portion of non-metazoan taxonomic assignments (Aylagas et al., 2018; Wangensteen et al., 2018). We tested this for primer set A, by blasting all ASVs that were unassigned after Midori (1471 ASVs) against the nt database and found only 45 ASVs with a non-metazoan hit. It remains unclear whether the remaining unassigned ASVs represent unknown diversity, genetic noise e.g. created by PCR and/or sequencing errors, nuclear pseudogenes, or aspecific amplification of other genomic regions.

The absence of morphological species in DNA metabarcoding datasets is not necessarily linked to the reference databases used for taxonomic assignment

Importantly, within the metazoan diversity DNA metabarcoding was not able to detect all morphological species, despite the fact that our reference database included 51 out of the 57 morphospecies. Our results illustrate that the reference database is not the only cause of the low number of ASVs with taxonomic assignments. When including all biological replicates, our best performing primer set (A) was unable to detect 19 out of the 59 morphological species. Only six of these 19 species did not have a reference sequence, indicating that other factors than the reference database are influencing species detection in metabarcode datasets. First, the wetlab procedure can lead to missing species because of differences in efficiency of DNA extraction between species or because primers insufficiently match with the COI gene of those species. All 19 species are relatively large animals, and for several species more than one individual was found in a particular sample. Several species have soft tissues, it is therefore unlikely that DNA extraction would be problematic for these species. Eleven species belonged to the Polychaeta, a class characterized by high species and sequence diversity in the COI gene (Carr, 2012). It is therefore likely that for these species, the primers were not a good match. This is further strengthened by the fact that for six polychaete species voucher specimens were available and good DNA was extracted, while no PCR product or bad Sanger sequences were obtained. This illustrates the great benefit of primer free methods for biodiversity assessment, although these are at this point more expensive than DNA metabarcoding (Giebner et al., 2020). Second, taxonomic assignment through the RDP classifier may be inefficient. One species, Acrocnida brachiata, was identified using the Midori reference dataset which contains $151 \mathrm{COI}$ sequences of this species, including a sequence that is identical to our own reference sequence. It is known that the content and size of the training set strongly impacts taxonomic assignment with RDP Classifier (Ritari et al., 2015). Finally, the morphological identification by our experts may have been incorrect. However, the morpho-taxonomic analyses of macrobenthos is under accreditation (accreditation certificate nr. 315-TEST, following NBN EN ISO/IEC 17025:2005) and the two experts that have conducted the morphological identification have very low misidentification rates (at most 3 taxa have been misidentified in a sample that underwent quality control over the last 9 years), suggesting that misidentification can only have a minor impact on our results. 


\section{DNA metabarcoding of macrobenthic communities in the North Sea is most comparable with}

morphology based analyses when using the Leray primer set

There is considerable support for the use of the mitochondrial COI gene to assess metazoan diversity from bulk samples (Andujar et al., 2018; Leray \& Knowlton, 2015; Lobo et al., 2017), yet finding the best primer set for a specific target group and study requires empirical data rather than relying on general rule of thumbs (Alberdi et al., 2018). In a study comparing the performance of five primer sets on mock communities of marine macrobenthos using the 454 sequencing platform, primer set $\mathrm{E}$ was amongst the best primer sets in terms of detecting many species (Hollatz et al., 2017). The amplification of the 3' region of the Folmer region has resulted in better detection of marine macroinvertebrates than the full length Folmer fragment (Aylagas, Borja, et al., 2016; Hollatz et al., 2017; Lobo et al., 2017). In silico testing showed that the primer sets in the 3 ' region generally detected almost twice the amount of species compared to the primer set in the 5' region (Table 1), which was confirmed by our wet lab results: primer sets amplifying the 3 ' region outperformed primer set D in recovering macrobenthic species. Our data clearly show that primer sets used by Leray et al. (2013) (Primer set A) and Lobo et al. (2013) (Primer set E) outcompete the other three primer sets in the number of high quality and non-chimeric reads (ESM Fig 1). Both primer sets amplify exactly the same fragment of the COI gene, but the reverse primer of primer set A has a higher degeneracy than the reverse primer of primer set E (Table 1). A higher degeneracy leads to a better performance of COI primers for insect communities (Marquina, Andersson, \& Ronquist, 2019). Based on the wetlab results, primer set A outperformed primer set $\mathrm{E}$ at various levels: 1/ twice the amount of sequences received taxonomic assignment (22.6 vs $10.9 \%$ ); 2 / the best correspondence with morphological species numbers in the different locations was obtained (ESM Fig 4) and 3/ the highest number of morphologically identified species was detected with primer set A (Fig 3). Finally, the Leray primer set was able to differentiate the four macrobenthic communities. Consequently, for monitoring studies targeting marine macrobenthic communities the use of the Leray primer set is recommended.

Metabarcode studies typically detect a higher number of species compared to morphology based analyses (Elbrecht, Vamos, et al., 2017; Lobo et al., 2017), which has been explained by the fact that specimens are not lost during the sorting process and also small pieces of animals - which are morphologically not identifiedare included in the bulk DNA and eDNA. In addition, metabarcode studies have reported the detection of species that were lacking in the morphological analyses from the same samples (Aylagas, Borja, et al., 2016). This was also the case for our study, but in contrast to what we expected, less species were detected using DNA metabarcoding (Fig 3): for the four samples that were processed both morphologically and with DNA metabarcoding, 57 species were morphologically identified, while 52 and 45 species were identified with the bulk DNA and eDNA, respectively. This may be partly explained by the reference database and algorithm used to assign taxonomy: 51 and 76 species for the bulk and eDNA, respectively, were identified when using the MIDORI reference database, while 60 and 103 species for the bulk and eDNA, respectively, were identified when using Blast and the NCBI database. The latter procedure was also used by Lobo et al. (2017) and Elbrecht, Vamos, et al. (2017).

Bulk DNA samples detect more macrobenthos species than eDNA from the ethanol preservative and are not affected by morphological traits of the species

The characterization of macrobenthos communities by DNA metabarcoding the ethanol preservative instead of bulk specimens would greatly speed up processing time of samples in the laboratory since the decantation and sorting step can be avoided. The use of the ethanol preservative to reliably characterize macroinvertebrate diversity and composition has been demonstrated for fresh water samples (Martins et al., 2019). Our results show that the number of morphological species detected in the ethanol preservative of each sample is lower compared to the bulk DNA samples (ESM Fig 4), which has also been observed for insect communities (Zenker, Specht, \& Fonseca, 2020) and for freshwater benthic communities (Hajibabaei et al., 2019). Substantial differences between both sample types have been observed with almost no shared species between bulk DNA and eDNA (Marquina, Esparza-Salas, et al., 2019). Our results also show significant differences between communities from the bulk and ethanol preservative (Table 2), but many species were detected in 
both sample types. The 15 species that were exclusively detected in the ethanol preservative were mainly pelagic species. In view of the low read numbers allocated to these species (at most 153 reads) these DNA molecules are most likely "contaminant" DNA molecules that were absorbed to the organic debris. Our results show that morphological traits of macrobenthos species can explain their detection in the eDNA from the ethanol preservative. Species with soft body skeleton and large body size showed higher probability of being detected in the eDNA fraction, while these traits did not strongly impact the detection in bulk DNA samples (Fig 5). The latter illustrates that mixing or grinding the samples before DNA extraction greatly increases the detection of a variety of species in bulk DNA samples.

\section{Towards standardization of DNA metabarcoding for marine environmental monitoring}

A European scale monitoring method has been identified as a science priority need to support benthic ecosystem assessments (Van Hoey et al., 2019). Biotic indexes inferred from DNA based taxonomic assignments are comparable to indexes derived from morphological identifications (Aylagas et al., 2018; Elbrecht, Vamos, et al., 2017; Lobo et al., 2017). When using DNA metabarcoding for monitoring, a standardized method that compares best with traditional morphological analyses in order not to break up the existing traditional monitoring time series is preferable. Our results show that bulk samples with use of the Leray primer set would be the method of choice for benthic monitoring in the North Sea. The next step is now to show case the repeatability and robustness of DNA metabarcoding for European legislation such as the Marine Strategy Framework Directive which may be achieved through the organization of a ring test in which the same samples are processed by different laboratories. Finally, our results show that complete reference sequence databases will not be the holy grail to come to $100 \%$ comparability with morpho-taxonomic datasets, but at this point the reference databases are our only link with the ecological and biological information imbedded in the Linnean system. As such, collaboration with taxonomic experts to further populate the reference database remain highly recommended (Porter \& Hajibabaei, 2018) to benefit as much as possible from the inclusion of DNA metabarcoding in monitoring studies.

\section{CONCLUSION}

DNA metabarcoding is a promising and cost-efficient tool for monitoring benthic communities in the marine environment. In view of the large taxonomic and phylogenetic diversity in marine communities (Appeltans et al., 2012; Mora, Tittensor, Adl, Simpson, \& Worm, 2011) and differences in benthic communities between geographic realms, optimisation of the protocol for the taxonomic group and geographic region at hand are necessary. By aiming for the detection of the highest number of known morphological species in a given area, a link with long time series of monitoring data based on morpho-taxonomy can be made. Our results demonstrate the impact of primer choice and DNA source when using the mitochondrial COI gene to assess macrobenthic species diversity. We show that the probability of detecting macrobenthos species is linked to morphological traits and this detection is most robust and most comparable with morphological results when using the Leray primer set and bulkDNA as template for DNA metabarcoding.

\section{ACKNOWLEDGEMENTS}

Funding for this research was received through the Sand Fund of the Federal Public Service Economy and GEANS- Genetic tools for Ecosystem health Assessment in the North Sea region, an Interreg project supported by the North Sea Programme of the European Regional Development Fund of the European Union. Shiptime on RV Belgica was provided by BELSPO and RBINS-OD Nature. We thank the crew for the logistic support during sampling.

\section{DATA ACCESSIBILITY STATEMENT}

The data that support the findings of this study will be openly available in the following repositories: 1) the newly generated COI sequences, along with the metadata, digital pictures and ab1 files have been uploaded to BOLD Systems v4 under the project ILGEA at http://www.boldsystems.org/; 2) The raw Illumina Miseq data with the corresponding metadata have been uploaded to the SRA under submission SUB1132638 at https://www.ncbi.nlm.nih.gov/; 3) R scripts will be made available through github after 
acceptance. ([dataset] Derycke, 2020)

\section{AUTHOR CONTRIBUTIONS}

Sofie Derycke designed the research, analyzed and interpreted the data and wrote the paper

Sara Maes performed the labwork, submitted sequences to Bold and wrote the manuscript

Laure Van den Bulcke conducted labwork

Joran Vanhollebeke wrote the $\mathrm{R}$ scripts and created the Github page

Jan Wittoeck identified the macrobenthos morphologically

Hans Hillewaert vouchered the specimens prior to barcoding

Bart Ampe helped in statistical analyses, interpreted statistical results and critically reviewed the manuscript

Annelies Haegeman helped in designing the study, interpreted the data and critically reviewed the manuscript

Kris Hostens provided funding for the research, designed the study and interpreted the data

Annelies Debacker provided funding for the research, interpreted the data and wrote the manuscript

\section{REFERENCES}

[dataset] Derycke, S. e. a. (2020). Scripts and links to sequence data from the paper "Optimisation of metabarcoding for monitoring marine macrobenthos: primer choice and morphological traits determine species detection in bulkDNA and eDNA from the ethanol preservative".

Alberdi, A., Aizpurua, O., Gilbert, M. T. P., \& Bohmann, K. (2018). Scrutinizing key steps for reliable metabarcoding of environmental samples. Methods in Ecology and Evolution, 9 (1), 134-147. doi:10.1111/2041210x.12849

Andujar, C., Arribas, P., Yu, D. W., Vogler, A. P., \& Emerson, B. C. (2018). Why the COI barcode should be the community DNA metabarcode for the metazoa. Molecular Ecology, 27 (20), 3968-3975. doi:10.1111/mec.14844

Appeltans, W., Ahyong, S. T., Anderson, G., Angel, M. V., Artois, T., Bailly, N., . . . Costello, M. J. (2012). The Magnitude of Global Marine Species Diversity. Current Biology, 22 (23), 2189-2202. doi:10.1016/j.cub.2012.09.036

Aylagas, E., Borja, A., Irigoien, X., \& Rodriguez-Ezpeleta, N. (2016). Benchmarking DNA Metabarcoding for Biodiversity-Based Monitoring and Assessment. Frontiers in Marine Science, 3. doi:10.3389/fmars.2016.00096

Aylagas, E., Borja, A., Muxika, I., \& Rodriguez-Ezpeleta, N. (2018). Adapting metabarcoding-based benthic biomonitoring into routine marine ecological status assessment networks. Ecological Indicators, 95 , 194-202. doi:10.1016/j.ecolind.2018.07.044

Aylagas, E., Mendibil, I., Borja, A., \& Rodriguez-Ezpeleta, N. (2016). Marine Sediment Sample Preprocessing for Macroinvertebrates Metabarcoding: Mechanical Enrichment and Homogenization. Frontiers in Marine Science, 3 . doi:10.3389/fmars.2016.00203

Barbier, E. B. (2017). Marine ecosystem services. Current Biology, 27 (11), R507-R510. doi:10.1016/j.cub.2017.03.020

Bates, D., Maechler, M., Bolker, B. M., \& Walker, S. C. (2015). Fitting Linear Mixed-Effects Models Using lme4. Journal of Statistical Software, 67 (1), 1-48. doi:10.18637/jss.v067.i01 
Benjamini, Y., \& Hochberg, Y. (1995). Controlling the false discovery rate - A practical and powerful approach to multiple testingJournal of the Royal Statistical Society Series B-Methodological, 57 (1), 289300.

Bolger, A. M., Lohse, M., \& Usadel, B. (2014). Trimmomatic: a flexible trimmer for Illumina sequence data. Bioinformatics, 30 (15), 2114-2120. doi:10.1093/bioinformatics/btu170

Borja, A., Elliott, M., Snelgrove, P. V. R., Austen, M. C., Berg, T., Cochrane, S., . . Wilson, C. (2016). Bridging the Gap between Policy and Science in Assessing the Health Status of Marine Ecosystems.Frontiers in Marine Science, 3 . doi:10.3389/fmars.2016.00175

Braukmann, T. W. A., Ivanova, N. V., Prosser, S. W. J., Elbrecht, V., Steinke, D., Ratnasingham, S., . . Hebert, P. D. N. (2019). Metabarcoding a diverse arthropod mock community. Molecular Ecology resources, 19 (3), 711-727. doi:10.1111/1755-0998.13008

Breine, N. T., De Backer, A., Van Colen, C., Moens, T., Hostens, K., \& Van Hoey, G. (2018). Structural and functional diversity of soft-bottom macrobenthic communities in the Southern North Sea. Estuarine Coastal and Shelf Science, 214 , 173-184. doi:10.1016/j.ecss.2018.09.012

Brusca, R. C., \& Brusca, G. J. (2002). Invertebrates (Second edition ed.). USA: Sinauer Associated Inc.

Callahan, B. J., McMurdie, P. J., Rosen, M. J., Han, A. W., Johnson, A. J. A., \& Holmes, S. P. (2016). DADA2: High-resolution sample inference from Illumina amplicon data. Nature Methods, 13 (7), 581-+. doi:10.1038/nmeth.3869

Carr, C. (2012). Polychaete diversity and distribution patterns in Canadian marine waters. Marine Biodiversity, 42 (2), 93-107. doi:10.1007/s12526-011-0095-y

Conway, J. R., Lex, A., \& Gehlenborg, N. (2017). UpSetR: an R package for the visualization of intersecting sets and their properties.Bioinformatics, 33 (18), 2938-2940. doi:10.1093/bioinformatics/btx364

Degraer, S., Verfaillie, E., Willems, W., Adriaens, E., Vincx, M., \& Van Lancker, V. (2008). Habitat suitability modelling as a mapping tool for macrobenthic communities: An example from the Belgian part of the North Sea. Continental Shelf Research, 28 (3), 369-379. doi:10.1016/j.csr.2007.09.001

Dixon, P. (2003). VEGAN, a package of R functions for community ecology.Journal of Vegetation Science, 14 (6), 927-930. doi:10.1111/j.1654-1103.2003.tb02228.x

Douvere, F., Maes, F., Vanhulle, A., \& Schrijvers, J. (2007). The role of marine spatial planning in sea use management: The Belgian case.Marine Policy, 31 (2), 182-191. doi:10.1016/j.marpol.2006.07.003

Duncan, C., Thompson, J. R., \& Pettorelli, N. (2015). The quest for a mechanistic understanding of biodiversity-ecosystem services relationships. Proceedings of the Royal Society B-Biological Sciences, 282 (1817). doi:10.1098/rspb.2015.1348

Elbrecht, V., \& Leese, F. (2017). Validation and Development of COI Metabarcoding Primers for Freshwater Macroinvertebrate Bioassessment.Frontiers in Environmental Science, 5 . doi:10.3389/fenvs.2017.00011

Elbrecht, V., Peinert, B., \& Leese, F. (2017). Sorting things out: Assessing effects of unequal specimen biomass on DNA metabarcoding.Ecology and Evolution, 7 (17), 6918-6926. doi:10.1002/ece3.3192

Elbrecht, V., Vamos, E. E., Meissner, K., Aroviita, J., \& Leese, F. (2017). Assessing strengths and weaknesses of DNA metabarcoding-based macroinvertebrate identification for routine stream monitoring. Methods in Ecology and Evolution, 8 (10), 1265-1275. doi:10.1111/2041-210x.12789

Elliott, S. A. M., Guerin, L., Pesch, R., Schmitt, P., Meakins, B., Vina-Herbon, C., . . Serrano, A. (2018). Integrating benthic habitat indicators: Working towards an ecosystem approach. Marine Policy, 90 , 88-94. doi:10.1016/j.marpol.2018.01.003 
Ewels, P., Magnusson, M., Lundin, S., \& Kaller, M. (2016). MultiQC: summarize analysis results for multiple tools and samples in a single report. Bioinformatics, 32 (19), 3047-3048. doi:10.1093/bioinformatics/btw354

Ficetola, G. F., Coissac, E., Zundel, S., Riaz, T., Shehzad, W., Bessiere, J., . . Pompanon, F. (2010). An In silico approach for the evaluation of DNA barcodes. BMC Genomics, 11 . doi:10.1186/1471-2164-11-434

Folmer, O., Black, M., Hoeh, W., Lutz, R., \& R, V. (1994). DNA primers for amplification of mitochondrial cytochrome c oxidase subunit I from diverse metazoan invertebrates. Molecular Marine Biology and Biotechnology, 3 , 294-299.

Fox, J., \& Weisberg, S. (2019). An $\{R\}$ Companion to Applied Regression. Thousand Oaks, CA: Sage.

Gauthier, M., Konecny-Dupre, L., Nguyen, A., Elbrecht, V., Datry, T., Douady, C., \& Lefebure, T. (2020). Enhancing DNA metabarcoding performance and applicability with bait capture enrichment and DNA from conservative ethanol. Molecular Ecology resources, 20 (1), 79-96. doi:10.1111/1755-0998.13088

Giebner, H., Langen, K., Bourlat, S. J., Kukowka, S., Mayer, C., Astrin, J. J., . . Fonseca, V. G. (2020). Comparing diversity levels in environmental samples: DNA sequence capture and metabarcoding approaches using 18S and COI genes. Molecular Ecology resources . doi:10.1111/1755-0998.13201

Goodwin, K. D., Thompson, L. R., Duarte, B., Kahlke, T., Thompson, A. R., Marques, J. C., \& Cacador, I. (2017). DNA Sequencing as a Tool to Monitor Marine Ecological Status. Frontiers in Marine Science, 4 . doi:10.3389/fmars.2017.00107

Hajibabaei, M., Porter, T. M., Robinson, C. V., Baird, D. J., Shokralla, S., \& Wright, M. T. G. (2019). Watered-down biodiversity? A comparison of metabarcoding results from DNA extracted from matched water and bulk tissue biomonitoring samples. PLoS ONE, 14 (12). doi:10.1371/journal.pone.0225409

Halpern, B. S., Longo, C., Lowndes, J. S. S., Best, B. D., Frazier, M., Katona, S. K., . . . Selig, E. R. (2015). Patterns and Emerging Trends in Global Ocean Health. PLoS ONE, 10 (3). doi:10.1371/journal.pone.0117863

Hollatz, C., Leite, B. R., Lobo, J., Froufe, H., Egas, C., \& Costa, F. O. (2017). Priming of a DNA metabarcoding approach for species identification and inventory in marine macrobenthic communities. Genome, 60 (3), 260-271. doi:10.1139/gen-2015-0220

Kebschull, J. M., \& Zador, A. M. (2015). Sources of PCR-induced distortions in high-throughput sequencing data sets. Nucleic Acids Research, 43 (21). doi:10.1093/nar/gkv717

Lenth, R. (2020). emmeans: Estimated Marginal Means, aka Least-Squares Means. R package version 1.4.7.

Leray, M., \& Knowlton, N. (2015). DNA barcoding and metabarcoding of standardized samples reveal patterns of marine benthic diversity.Proceedings of the National Academy of Sciences of the United States of America, 112 (7), 2076-2081. doi:10.1073/pnas.1424997112

Leray, M., Yang, J. Y., Meyer, C. P., Mills, S. C., Agudelo, N., Ranwez, V., . . . Machida, R. J. (2013). A new versatile primer set targeting a short fragment of the mitochondrial COI region for metabarcoding metazoan diversity: application for characterizing coral reef fish gut contents. Frontiers in Zoology, 10 . doi:10.1186/1742-9994-10-34

Lobo, J., Costa, P. M., Teixeira, M. A. L., Ferreira, M. S. G., Costa, M. H., \& Costa, F. O. (2013). Enhanced primers for amplification of DNA barcodes from a broad range of marine metazoans. BMC Ecology, 13 . doi:10.1186/1472-6785-13-34

Lobo, J., Shokralla, S., Costa, M. H., Hajibabaei, M., \& Costa, F. O. (2017). DNA metabarcoding for highthroughput monitoring of estuarine macrobenthic communities. Sci Rep, 7 . doi:10.1038/s41598-017-15823-6

Macheriotou, L., Guilini, K., Bezerra, T. N., Tytgat, B., Nguyen, D. T., Nguyen, T. X. P., . . Derycke, S. (2019). Metabarcoding free-living marine nematodes using curated $18 \mathrm{~S}$ and CO1 reference sequence databases 
for species-level taxonomic assignments. Ecology and Evolution, 9 (3), 1211-1226. doi:10.1002/ece3.4814

Machida, R. J., Leray, M., Ho, S. L., \& Knowlton, N. (2017). Data Descriptor: Metazoan mitochondrial gene sequence reference datasets for taxonomic assignment of environmental samples. Scientific Data, 4 . doi:10.1038/sdata.2017.27

Marquina, D., Andersson, A. F., \& Ronquist, F. (2019). New mitochondrial primers for metabarcoding of insects, designed and evaluated using in silico methods. Molecular Ecology resources, 19 (1), 90-104. doi:10.1111/1755-0998.12942

Marquina, D., Esparza-Salas, R., Roslin, T., \& Ronquist, F. (2019). Establishing arthropod community composition using metabarcoding: Surprising inconsistencies between soil samples and preservative ethanol and homogenate from Malaise trap catches. Molecular Ecology resources, 19 (6), 1516-1530. doi:10.1111/17550998.13071

Martinez Arbizu, P. (2019). pairwiseAdonis: Pairwise multilevel comparison using adonis. (Version R package version 0.3). Retrieved from https://github.com/pmartinezarbizu/pairwiseAdonis

Martins, F. M. S., Galhardo, M., Filipe, A. F., Teixeira, A., Pinheiro, P., Pauperio, J., . . Beja, P. (2019). Have the cake and eat it: Optimizing nondestructive DNA metabarcoding of macroinvertebrate samples for freshwater biomonitoring. Molecular Ecology resources, 19 (4), 863-876. doi:10.1111/1755-0998.13012

Mora, C., Tittensor, D. P., Adl, S., Simpson, A. G. B., \& Worm, B. (2011). How Many Species Are There on Earth and in the Ocean? PLoS Biology, 9 (8). doi:10.1371/journal.pbio.1001127

Motokawa, T. (1984). CONNECTIVE-TISSUE CATCH IN ECHINODERMS.Biological Reviews of the Cambridge Philosophical Society, 59 (2), 255-270. doi:10.1111/j.1469-185X.1984.tb00409.x

Pawlowski, J., Kelly-Quinn, M., Altermatt, F., Apotheloz-Perret-Gentil, L., Beja, P., Boggero, A., . . . Kahlert, M. (2018). The future of biotic indices in the ecogenomic era: Integrating (e) DNA metabarcoding in biological assessment of aquatic ecosystems. Science of the Total Environment, 637 , 1295-1310. doi:10.1016/j.scitotenv.2018.05.002

Porter, T. M., \& Hajibabaei, M. (2018). Automated high throughput animal CO1 metabarcode classification. Sci Rep, 8 . doi:10.1038/s41598-018-22505-4

Quinn, G., \& Keough, M. (2002). Experimental design and data analysis for biologists . New York: Cambridge University Press.

Ritari, J., Salojarvi, J., Lahti, L., \& de Vos, W. M. (2015). Improved taxonomic assignment of human intestinal $16 \mathrm{~S}$ rRNA sequences by a dedicated reference database. BMC Genomics, 16 . doi:10.1186/s12864015-2265-y

Solan, M., Cardinale, B. J., Downing, A. L., Engelhardt, K. A. M., Ruesink, J. L., \& Srivastava, D. S. (2004). Extinction and ecosystem function in the marine benthos. Science, 306 (5699), 1177-1180. doi: $10.1126 /$ science. 1103960

Team, R. C. (2019). R: A language and environment for statistical computing. Vienna, Austria: R Foundation for Statistical Computing. Retrieved from http://www.R-project.org

Van Hoey, G., Degraer, S., \& Vincx, M. (2004). Macrobenthic community structure of soft-bottom sediments at the Belgian Continental Shelf.Estuarine Coastal and Shelf Science, 59 (4), 599-613. doi:10.1016/j.ecss.2003.11.005

Van Hoey, G., Wischnewski, J., Craeymeersch, J., Dannheim, J., Enserink, L., Guerin, L., . . Birchenough, S. N. R. (2019). Methodological elements for optimising the spatial monitoring design to support regional benthic ecosystem assessments. Environmental Monitoring and Assessment, 191 (7). doi:10.1007/s10661019-7550-9 
Wang, Q., Garrity, G. M., Tiedje, J. M., \& Cole, J. R. (2007). Naive Bayesian classifier for rapid assignment of rRNA sequences into the new bacterial taxonomy. Applied and Environmental Microbiology, 73 (16), 5261-5267. doi:10.1128/aem.00062-07

Wangensteen, O. S., Palacin, C., Guardiola, M., \& Turon, X. (2018). DNA metabarcoding of littoral hardbottom communities: high diversity and database gaps revealed by two molecular markers. Peerj, 6 . doi:10.7717/peerj. 4705

Zenker, M. M., Specht, A., \& Fonseca, V. G. (2020). Assessing insect biodiversity with automatic light traps in Brazil: Pearls and pitfalls of metabarcoding samples in preservative ethanol. Ecology and Evolution, 10 (5), 2352-2366. doi:10.1002/ece3.6042

Zizka, V. M. A., Elbrecht, V., Macher, J. N., \& Leese, F. (2019). Assessing the influence of sample tagging and library preparation on DNA metabarcoding. Molecular Ecology resources, 19 (4), 893-899. doi:10.1111/17550998.13018

Zizka, V. M. A., Leese, F., Peinert, B., \& Geiger, M. F. (2019). DNA metabarcoding from sample fixative as a quick and voucher-preserving biodiversity assessment method. Genome, 62 (3), 122-136. doi:10.1139/gen2018-0048

\section{FIGURE LEGENDS}

Figure 1: Overview map with (A) location of the Belgian part of the North Sea (BPNS) in the wider North Sea and (B) sampling locations of this study in the BPNS. Colors indicate the distribution of the macrobenthic communities (after Degraer et al. 2008).

Figure 2: Percentage of ASVs found in ethanol (E) and bulk (B) samples that received taxonomic assignment at the different taxonomic levels for each primer set using the unrarified datasets.

Figure 3: The number of unique and shared species between morphological, bulk DNA and eDNA from the ethanol preservative datasets for each of the five primer sets (A, B, C, D and E). Datasets are based on the four samples that were identified morphologically to allow a one-on-one comparison.

Figure 4: Multidimensional scaling plots based on Bray Curtis dissimilarity index for each primer set (A,B,C,D and E). Colors indicate the four locations (120: red, 330: blue, 840:green and ZVL: purple), full circles indicate bulk DNA and open triangles eDNA samples from the ethanol preservative.

Figure 5: Effect of sample type, body size and body skeleton on the probability of detecting macrobenthos species in bulk DNA (B) or eDNA from the ethanol preservative (E) samples in relation to body size (A) and body skeleton (B). Probabilities are based on the generalized linear mixed effects model in Table 3.

\section{TABLES}

Table 1. Overview of the COI primersets used for in silico testing against the Midori marine database. "Code" refers to the six primer sets that were tested in the wetlab. The five primer sets with succesful PCR bands are indicated with A,B,C,D,E. Forward and reverse sequences of the primers and their original reference are listed. "Number of morphological families/species' refers to the number of families/species that were identifed morphologically and that were also retrieved in the in silico test with EcoPCR. "Number of species in Midori database" refers to the number of species from the Midroi database that were picked up in the in silico test for each primer set.

\section{Hosted file}

image1.emf available at https://authorea.com/users/349000/articles/474144-optimisation-ofmetabarcoding-for-monitoring-marine-macrobenthos-primer-choice-and-morphological-traitsdetermine-species-detection-in-bulkdna-and-edna-from-the-ethanol-preservative

Table 2. PERMANOVA and Permdisp results based on 9999 permutations for each of the five primersets using Bray-Curtis distance or the Jaccard index. 


\section{Hosted file}

image2.emf available at https://authorea.com/users/349000/articles/474144-optimisation-ofmetabarcoding-for-monitoring-marine-macrobenthos-primer-choice-and-morphological-traitsdetermine-species-detection-in-bulkdna-and-edna-from-the-ethanol-preservative

Table 3. The effect of Sample type, Body size and Body skeleton on the probability of detecting a macrobenthos species. Type III tests of the fixed effects of the final generalized linear mixed effects model are shown.

\section{Hosted file}

image3.emf available at https://authorea.com/users/349000/articles/474144-optimisation-ofmetabarcoding-for-monitoring-marine-macrobenthos-primer-choice-and-morphological-traitsdetermine-species-detection-in-bulkdna-and-edna-from-the-ethanol-preservative 


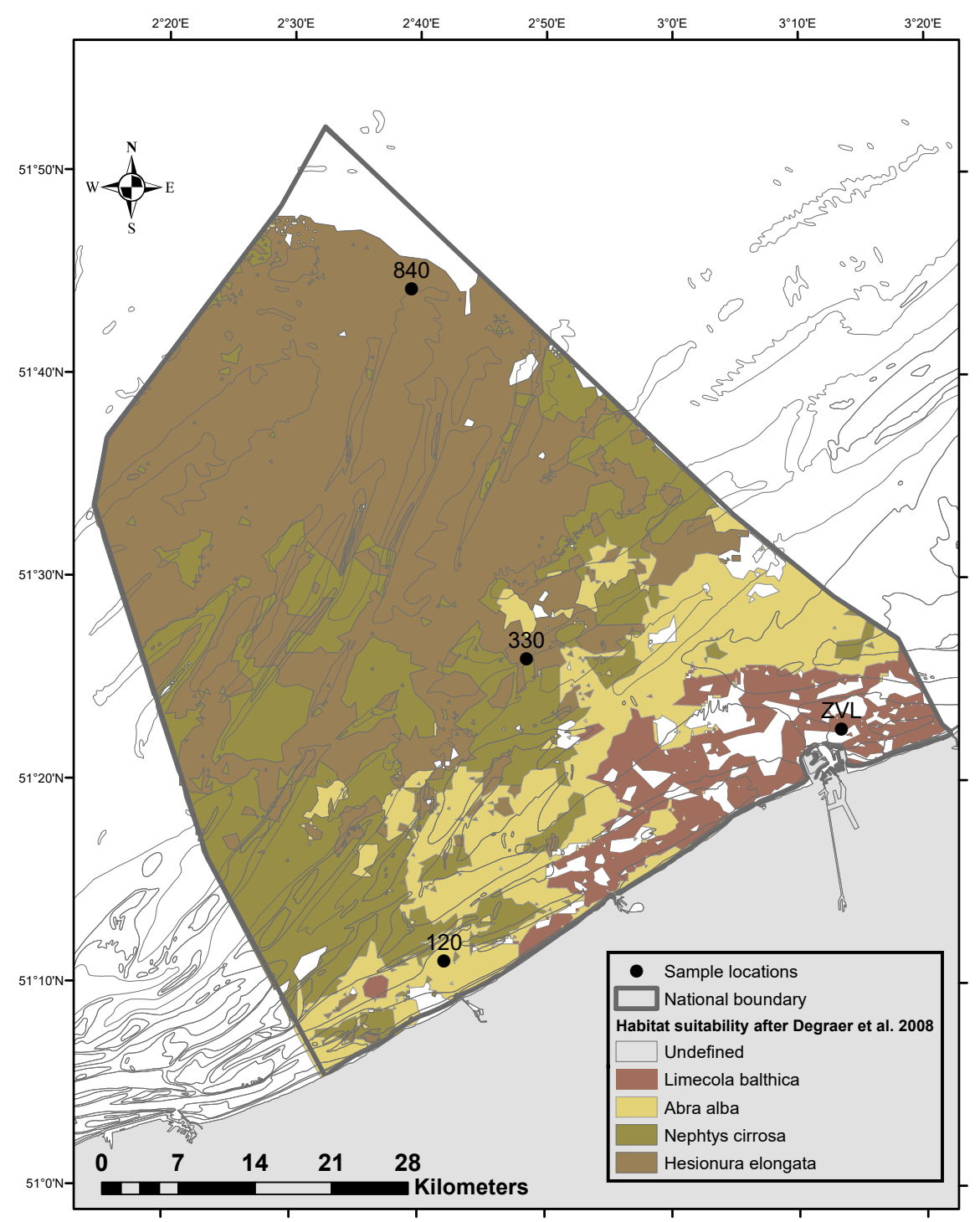




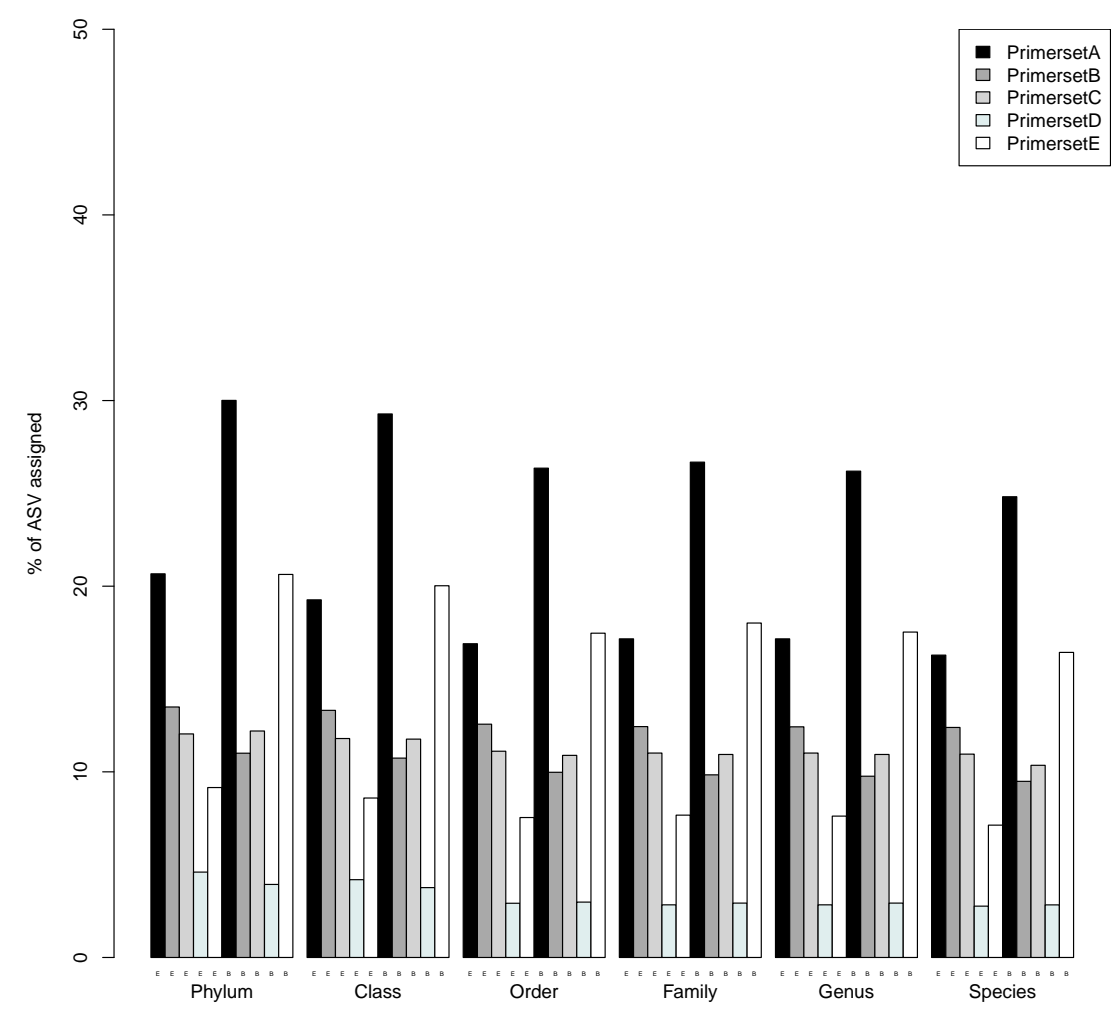


A

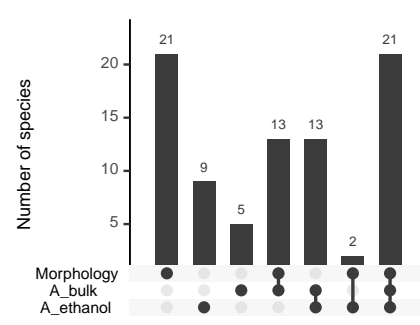

C

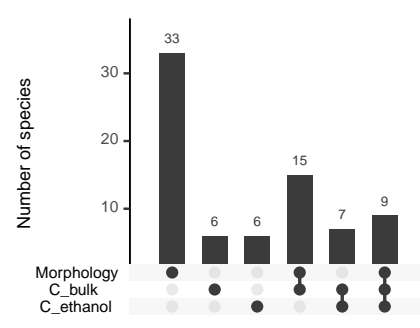

B

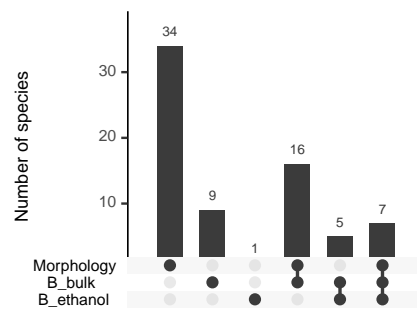

D

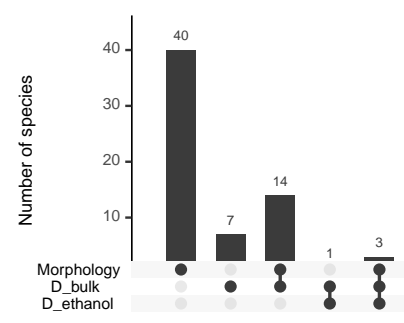

E
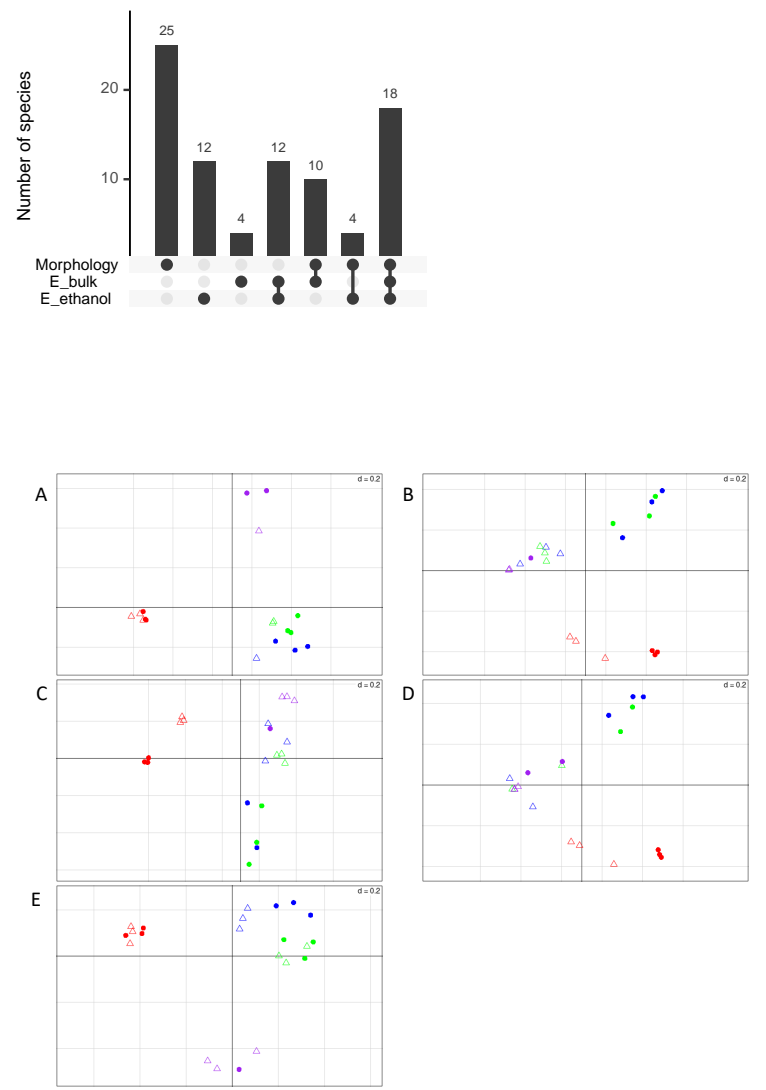
A

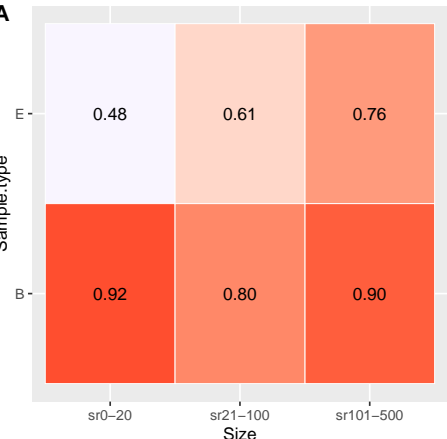

B

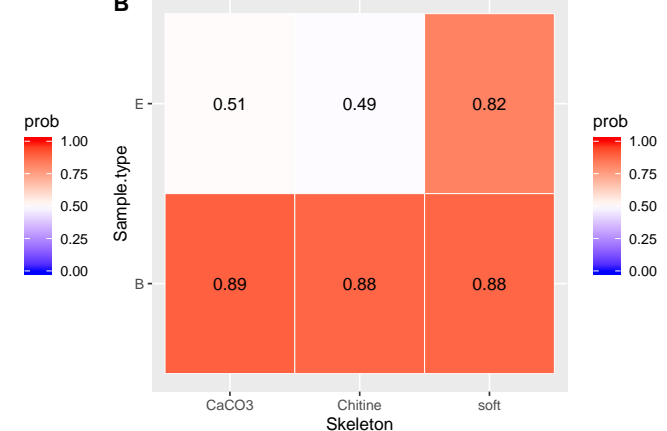

